Cleide Mayra Menezes Lima

\title{
Um estudo sobre o método Mínimos Quadrados Móveis por Aproximações Iteradas
}

Dissertação apresentada como requisito parcial para obtenção do grau de Mestre pelo Programa de Pós-graduação em Matemática do Departamento de Matemática da PUC-Rio

Orientador: Prof. Hélio Côrtes Vieira Lopes

Rio de Janeiro

Setembro de 2009 


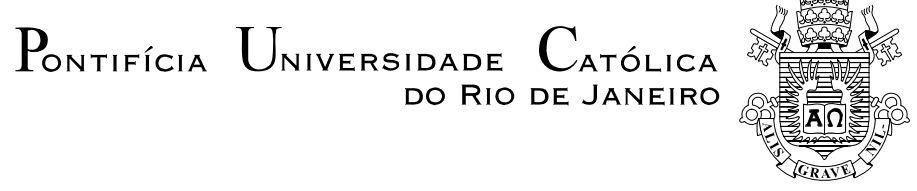

Cleide Mayra Menezes Lima

\section{Um estudo sobre o método Mínimos} Quadrados Móveis por Aproximações Iteradas

Dissertação apresentada como requisito parcial para obtenção do grau de Mestre pelo Programa de PósGraduação em Matemática do Departamento de Matemática do Centro Técnico Científico da PUC-Rio. Aprovada pela Comissão Examinadora abaixo assinada.

Prof. Hélio Côrtes Vieira Lopes Orientador Departamento de Matemática - PUC-Rio

Prof. Thomas Lewiner Departamento de Matemática - PUC-Rio

Profa. Dirce Uesu Pesco Instituto de Matemática - UFF

Prof. Sinésio Pesco Departamento de Matemática - PUC-Rio

Prof. José Eugenio Leal Coordenador Setorial do Centro Técnico Científico - PUC-Rio 
Todos os direitos reservados. É proibida a reprodução total ou parcial do trabalho sem autorização da autora, do orientador e da universidade.

\section{Cleide Mayra Menezes Lima}

Graduou-se em bacharelado pela Matemática pela Universidade Federal do Piauí em 2003.

Ficha Catalográfica

Cleide Mayra Menezes Lima

Um estudo sobre o método Mínimos Quadrados Móveis por Aproximações Iteradas / Cleide Mayra Menezes Lima; orientador: Hélio Côrtes Vieira Lopes. - Rio de Janeiro: PUC-Rio, Departamento de Matemática, 2009.

v., 60 f: il. ; $29,7 \mathrm{~cm}$

1. Dissertação (Mestrado em Matemática) - Pontifícia Universidade Católica do Rio de Janeiro, Departamento de Matemática.

Inclui referências bibliográficas.

1. Matemática - Tese. 2. Interpolação. 3. Aproximação. 4. Modelagem Geométrica. I. Lopes, H.. II. Pontificia Universidade Católica do Rio de Janeiro. Departamento de Matemática. III. Título.

CDD: 510 
A Deus pelas bençãos que tem derramado em minha vida. Aos meus pais pelos ensinamentos, dedicação e carinho. Aos meus irmãos pelo apoio e aos meus sobrinhos Bianca e Arthur. 


\section{Agradecimentos}

A Deus, por me oferecer saúde, disposição, discernimento e por colocar várias pessoas maravilhosas na minha vida, além de me fornecer inúmeras oportunidades. A Ele, só tenho a agradecer por tudo.

Aos meus pais Cleidomir e Maria de Jesus pelos ensinamentos, dedicação e carinho. Aos meus irmãos Janery, Janayna, Jacqueline e Júnior, e também aos meus queridos sobrinhos Bianca e Arthur.

À minha amiga Valmária, seu esposo Fernando, e à sua família, pelo carinho, apoio e incentivo.

À família Bandeira: Mônica, Luizinho e aos seus filhos Matheus, Felipe e Nathan pelo carinho.

Quero expressar também minha gratidão ao programa de Pós-Graduação em Matemática da PUC-RJ, pela oportunidade que me foi dada de fazer parte do programa e em especial ao meu professor e orientador Hélio Côrtes Vieira Lopes pela paciência, oportunidade e confiança.

Às secretárias, Creusa e Kátia, e aos auxiliares administrativos, Orlando e Otávio, pela amizade e competência.

Às amigas do Mestrado Joana, Marina, Mariana e Carla.

Quero agradecer também aos meus amigos: Fernando, Júnior, Fidel, Vinicius e Marcelo, pela enorme ajuda que me deram.

Aos amigos que aqui no Rio de Janeiro tive a oportunidade de conhecer: Clarice, Antônio, Marilane, Alexandre, Luzia e Ângelo.

À Igreja Batista de Betânia-RJ, por ser um canal de benção em minha vida.

À Pontifícia Universidade Católica do Rio de Janeiro (PUC-RJ) pela bolsa de isenção concedida.

À CAPES pelo apoio financeiro.

Aos amigos e professores da Universidade Federal do Piauí.

Em memória ao meu amigo Waguinho, que tive a oportunidade de conhecer e conviver na minha estada no Rio, e ao Renato que foi meu monitor no início do Mestrado. Saudades!

Enfim, a todos que me ajudaram, direta ou indiretamente, meus mais sinceros agradecimentos. 


\section{Resumo}

Lima, Cleide Mayra Menezes; Lopes, Hélio Côrtes Vieira (Orientador). Um estudo sobre o método Ménimos Quadrados Móveis por Aproximaçōes lteradas. Rio de Janeiro, 2009. 60p. Dissertação de Mestrado - Departamento de Matemática, Pontifícia Universidade Católica do Rio de Janeiro.

Esta dissertação tem por objetivo estudar un método para aproximação de dados esparços multivariados denominado o método Mínimos Quadrados Móveis por Aproximações Iteradas (Iterated Approximate Moving LeastSquare Approximation - IAMLS). Este método é baseado no método de interpolação por funções de base radial (RBF) e no método de aproximação AMLS. Mas diferentemente do mćtodo RBF, cle não requer a solução de um sistema de equações lineares. O método IAMLS no limite converge para o interpolante RBF sob certas condições.

\section{Palavras-chave}

Interpolação. Aproximação. Modelagem Geométrica. 


\section{Abstract}

Lima, Cleide Mayra Menezes; Lopes, Hélio Côrtes Vieira (Advisor). A study about the Iterated Approximated Moving Least Squares Method. Rio de Janeiro, 2009. 60p. MSC Dissertation - Departamento de Matemática, Pontifícia Universidade Católica do Rio de Janeiro.

The objective of his work is to study an approximation method for multivariate sparse data named Iterated Approximate Moving Least Square Approximation - IAMLS. This method is based on the Radial Basis Functions (RBF) interpolation method and on the AMLS approximation method. Differently from the RBF interpolation method, the IAMLS does not requires to solve a system of linear equations. The IAMLS method converges to the RBF interpolant under some conditions.

\section{Keywords}

Interpolation. Approximation. Geometric Modeling. 


\section{Sumário}

1 Introdução 12

2 Interpolação usando Funções de Base Radial 14

2.1 Introdução 14

2.2 O problema de interpolação de dados esparsos $\quad 16$

2.3 Interpolação RBF 17

$\begin{array}{ll}2.4 \text { Funções estritamente positivas definidas } & 17\end{array}$

$\begin{array}{ll}2.5 \text { Selecionando o melhor parâmetro de forma para RBF } & 18\end{array}$

3 Interpolação via Aproximação Iterada AMLS 21

3.1 Mínimos-quadrados-móveis aproximados - AMLS 21

3.2 Funções de base radial para o método IAMLS 23

3.3 O método IAMLS 25

4 Experimentos Numéricos $\quad 30$

$\begin{array}{ll}4.1 \text { Análise Unidimensional } & 30\end{array}$

$\begin{array}{ll}4.2 \text { Análise Bidimensional } & 40\end{array}$

5 Conclusão e trabalhos futuros $\quad 58$

Referências Bibliográficas $\quad 59$ 


\section{Lista de figuras}

2.1 Exemplos gráficos de funções de base radial. 20

3.1 Exemplos de funções Laguerre-Gaussianas. 28

3.2 Exemplos de funções Multiquádrica Inversas Generalizadas. 29

4.1 Gráfico da função de Franke 31

4.2 Análise do intervalo de convergência do método IAMLS com relação ao parâmetro $\epsilon$ utilizando uma um conjunto $\mathcal{S}$ com 30 pontos uniformemente espaçados e outro com 30 pontos não-uniformente espaçados.

4.3 Análise gráfica da convergência do Método IAMLS.

4.4 Análise gráfica da convergência do Método IAMLS usandos as estatísticas EQ, EQM, REQM e DP - gráficos à esquerda utilizam 1 iteração e à direita utlizam 3 iterações.

4.5 Gráficos que representam 100 pontos interpolados pelo Método RBF e IAMLS considerando uma amostra de 30 pontos igualmente espaçados.

4.6 Teste LOOCV para Método RBF.

4.7 Gráficos do EQ máximo e da REQM. 37

4.8 Análise da convergência do método IAMLS. 38

4.9 Gráficos do EQ máximo e da REQM para o método IAMLS. 39

4.10 Superfície topográfica do dado volcano disponível na plataforma R. 40

4.11 Análise do intervalo de convergência do método IAMLS com relação ao parâmetro $\epsilon$.

4.12 Gráficos do Teste LOOCV - "leave-one-out" cross validation e da Análise do Erro Máximo e REQM para as amostras de tamanho $N=20$ e $N=30$

4.13 Comparação visual da superfície verdadeira com a superfície interpolada - Método RBF.

4.14 Análise do EQ e REQM do Método IAMLS após $n$ iterações.

4.15 Comparação da Superfície original com a Superfície interpolada pelo Método RBF e pelo método IAMLS (após 25 iterações).

4.16 Análise de convergência do Método IAMLS para RBF usando as funções Laguerre Gaussiana e Multiquádrica Inversa Generalizada.

4.17 Análise do Método RBF através do teste LOOCV e estatísticas EQ, REQM e DP em relação ao EQM.

4.18 Superfícies interpoladas usando o Método RBF e a Função Laguerre-Gaussiana.

4.19 Gráficos do EQ e REQM.

4.20 Superfícies interpoladas pelo Método RBF e IAMLS (10iterações) usando a Função Laguerre-Gaussiana.

4.21 Análise do Método RBF.

4.22 Teste LOOCV para o Método RBF usando a função Multiquádrica Inversa Generalizada. 
4.23 Superfícies interpoladas usando o Método RBF e a Função Multiquádrica Inversa Genralizada.

4.24 Gráficos do EQ e REQM.

4.25 Superfícies interpoladas pelo Método RBF e IAMLS (10iterações) usando a Função Multiquádrica Inversa Generalizada. 


\section{Lista de tabelas}

2.1 Alguns tipos clássicos de funções de base radial.

3.1 Exemplos de função polinomial Laguerre generalizada de grau $d$ em $\mathbb{R}^{s}$.

3.2 Exemplos de funções multiquádricas inversas generalizadas.

4.1 Estatísticas de erros para o método RBF.

4.2 Estatísticas de erros para o método IAMLS

4.3 Estatísticas de erros para o método RBF.

4.4 Análise estatística da convergência do método IAMLS. 37

4.5 Método RBF

4.6 Análise da convergência do Método IAMLS para o RBF. 44

4.7 Análise do Método RBF usando a função Laguerre-Gaussina. 48

4.8 Análise da Convergência do Método IAMLS para o RBF usando a Função Laguerre-Gaussiana.

4.9 Análise do Método RBF usando a função Multiquádrica Inversa Generalizada.

4.10 Análise da Convergência do Método IAMLS para o RBF usando a Função Multiquádrica Inversa Generalizada. 